\title{
ARTINIAN SKEW GROUP RINGS ${ }^{1}$
}

\author{
JAE KEOL PARK
}

\begin{abstract}
Let $R$ be a ring with identity and let $\theta$ be a group homomorphism from a group $G$ to $\operatorname{Aut}(R)$, the group of automorphisms of $R$. We prove that skew group ring $R *_{\theta} G$ is right Artinian (resp., semiprimary, right perfect) if and only if $R$ is right Artinian (resp., semiprimary, right perfect) and the group $G$ is finite. Also semilocal skew group rings over fields are characterized.
\end{abstract}

1. Introduction. In [2] I. G. Connell has shown that the ordinary group ring $R[G]$ is right Artinian if and only if $R$ is right Artinian and $G$ is finite. Moreover, G. Renault [11] and S. M. Woods [12] have shown that the group ring $R[G]$ is right perfect if and only if $R$ is right perfect and $G$ is finite.

In general, this type of result is not true for crossed products. D. S. Passman [10, Proposition 4.2] has constructed an Artinian twisted group ring with an infinite group.

Joe W. Fisher has raised the question in the case of skew group rings, i.e., crossed products with trivial factor sets. The usual group ring techniques fail for skew group rings; however, in spite of this, we have still been able to show that the answer to his question is affirmative, i.e., a skew group ring is right Artinian (resp., semiprimary, right perfect) if and only if the coefficient ring is right Artinian (resp., semiprimary, right perfect) and the group is finite. In order to show that this kind of result does not extend beyond the perfect case, we give an example of a skew group ring which is semiperfect but the group is not finite. Also, in this paper, semilocal skew group rings over fields will be characterized.

2. Preliminaries. In this section some necessary definitions and lemmas will be introduced. Throughout this paper, $R$ denotes a ring with identity and $G$ denotes a group.

Let $\theta$ be a group homomorphism from $G$ to $\operatorname{Aut}(R)$, the group of automorphisms of the ring $R$. The skew group ring $R *_{\theta} G$ is defined as in [5] to be $R *_{\theta} G=\bigoplus \sum_{g \in G} R g$ with addition given componentwise and

Presented to the Society, May 18, 1978; received by the editors May 31, 1978 and, in revised form, August 18, 1978.

AMS (MOS) subject classifications (1970). Primary 16A26, 16A46, 16 A72.

Key words and phrases. Skew group rings, semilocal rings, perfect rings, semiperfect rings, semiprimary rings, crossed products, outer automorphism.

'This paper will form part of author's doctoral dissertation at the University of Cincinnati under the supervision of Professor Joe W. Fisher. 
multiplication given as follows: if $r, s \in R$ and $g, h \in G$, then $(r g)(s h)=$ $r s^{\theta(g)} g h$, where $s^{\theta(g)}$ is the image of $s$ under $\theta(g)$.

The ring $R$ is called right perfect [1] if $R$ is semilocal (i.e. $R / J(R)$ is Artinian) and the Jacobson radical $J(R)$ is right $T$-nilpotent, or equivalently, $R$ satisfies the descending chain condition on principal left ideals [1, Theorem 28.4 , p. 315]. The ring $R$ is called semiprimary [1] if $R$ is semilocal and $J(R)$ is nilpotent. Finally $R$ is called semiperfect [1] if $R$ is semilocal and every idempotent in $R / J(R)$ can be lifted to one in $R$.

We begin with the following well-known result.

Proposition 2.1. Let $S$ be a ring with identity and $R$ be a subring of $S$ with the same identity. If $R$ is a left (resp., right) $R$-direct summand of $S$, then for any right (resp., left) ideal $I$ of $R, I S \cap R=I$ (resp., $S I \cap R=I$ ).

Proof. Straightforward.

The usual group ring technique of obtaining $R$ as a homomorphic image of $R *{ }_{\theta} G$ via the augmentation map fails because of the skewing of the coefficients. The following lemmas overcome this difficulty.

LEMMA 2.2. If the skew group ring $R *{ }_{\theta} G$ is right Artinian (resp., right Noetherian, right perfect), then so is $R *{ }_{\theta} H$ for any subgroup $H$ of $G$. In particular, $R$ is right Artinian (resp., right Noetherian, right perfect).

Proof. Evident from Proposition 2.1.

LEMMA 2.3. Let $R$ be a ring with identity and $\theta$ be a group homomorphism from $G$ to $\operatorname{Aut}(R)$. And let $B$ be a subring of $R$ with the same identity such that $B$ is invariant under the action of $\theta(G)$. If $B$ is a left (resp., right) B-direct summand of $R$, then, for any right (resp., left) ideal $I$ of $B *{ }_{\sigma} G, I\left(R *{ }_{\theta} G\right) \cap$ $B *_{\sigma} G=I$ (resp., $\left.\left(R *_{\theta} G\right) I \cap B *_{\sigma} G=I\right)$, where $\sigma$ is the group homomorphism from $G$ to $\operatorname{Aut}(B)$ induced by $\theta$.

Proof. Let $B$ be a left $B$-direct summand of $R$ such that ${ }_{B} R={ }_{B} B \oplus_{B} C$ and let $G=\{e, g, h, \ldots\}$. Then $R *_{\theta} G=B *_{\sigma} G \oplus(e C \oplus g C \oplus h C$ $\bigoplus \ldots)$ and $e C \oplus g C \oplus h C \oplus \ldots$ is a left $B *{ }_{\sigma} G$-module. Therefore $B *_{\sigma} G$ is a left $B *{ }_{\sigma} G$-module direct summand of $R *_{\theta} G$. Hence, for any right ideal $I$ of $B *{ }_{\sigma} G, I\left(R *{ }_{\theta} G\right) \cap B *{ }_{\sigma} G=I$ by Proposition 2.1 .

Similarly for any left ideal $I$ of $B *{ }_{\sigma} G,\left(R *_{\theta} G\right) I \cap B *_{\sigma} G=I$ if $B$ is a right $B$-direct summand of $R$.

3. Main results. In this section, we establish our main theorems. We will start with the Artinian result. In order to do this, we will first consider a right Artinian skew group ring whose coefficient ring is primitive. Secondly, we allow the coefficient ring to be semiprimitive and, finally, we are able to handle the general case. 
Proposition 3.1. Let $R$ be a primitive ring and let $\theta$ be a group homomorphism from a group $G$ to $\operatorname{Aut}(R)$. If $R *_{\theta} G$ is right Artinian, then $G$ is finite.

Proof. Since $R *{ }_{\theta} G$ is right Artinian and $R$ is primitive, $R$ is a simple Artinian ring by Lemma 2.2. Thus the center $F$ of $R$ is a field. And since $F$ is invariant under the action of $\theta(G), F *{ }_{\sigma} G$ is right Artinian by Lemma 2.3, where $\sigma$ is the group homomorphism from $G$ to $\operatorname{Aut}(F)$ induced by $\theta$. Now let $F^{\sigma(G)}$ be the fixed subfield of $F$ under the action of $\sigma(G)$, that is, $F^{o(G)}=\{a$ $\in F: a^{\sigma(g)}=a$ for all $g$ in $\left.G\right\}$. Then by Lemma 2.3, we obtain that $F^{\sigma(G)}{ }_{s} G=F^{\sigma(G)}[G]$ (the ordinary group ring) is right Artinian, where $s$ is the group homomorphism from $G$ to $\operatorname{Aut}\left(F^{\sigma(G)}\right)$ induced by $\sigma$. Hence by Connell's result [2, Theorem 1], we get that $G$ is finite.

LEMMA 3.2. Let $R$ be a semiprimitive ring and $\theta$ be a group homomorphism from a group $G$ to $\operatorname{Aut}(R)$. If $R *_{\theta} G$ is right Artinian, then $G$ is finite.

Proof. Since $R *{ }_{\theta} G$ is right Artinian, $R$ is right Artinian by Lemma 2.2, and therefore $R$ is semiprimitive Artinian. Now let $R=R_{1} \oplus R_{2} \oplus \cdots \oplus$ $R_{n}$ be the decomposition into simple components and let $H=\operatorname{Ker} \theta$. Then, since $R *_{\theta} G$ is right Artinian, $R *_{\theta} H=R[H]$ (the ordinary group ring) is right Artinian by Lemma 2.2. Hence $H$ is finite by Connell's result [2, Theorem 1].

Now to prove that $G$ is finite, it will suffice to show that $\theta(G)$ is finite because $G / H \cong \theta(G) \subseteq \operatorname{Aut}(R)$. Then since $\theta(G)$ acts on $R=R_{1} \oplus R_{2}$ $\oplus \cdots \oplus R_{n}, \theta(G)$ permutes these factors $R_{i}$. Thus if $H_{1}=\{\theta(g) \in \theta(G)$ : $\left.R_{1}^{\theta(g)}=R_{1}\right\}$, then we see that $\left[\theta(G): H_{1}\right] \leqslant n$. Furthermore, by Lemmas 2.2 and 2.3, $R_{1} * H_{1}$ is right Artinian. It follows from Lemma 3.1 that $H_{1}$ is finite. Hence $\theta(G)$ is finite. Therefore $G$ is finite.

Now we are in a position to prove one of our main results.

THEOREM 3.3. Let $R$ be a ring and $\theta$ be a group homomorphism from a group $G$ to $\operatorname{Aut}(R)$. Then $R *_{\theta} G$ is right Artinian if and only if $R$ is right Artinian and the group $G$ is finite.

Proof. The sufficiency is evident. For the necessity, suppose that $R *_{\theta} G$ is right Artinian. Then by Lemma 2.2, $R$ is right Artinian. Let $J(R)$ denote the Jacobson radical of $R$. Then $\theta$ induces a group homomorphism $\sigma$ from $G$ to $\operatorname{Aut}(R / J(R))$ because the Jacobson radical $J(R)$ is obviously invariant under the action of $\theta(G)$. In this case $R / J(R) *_{\sigma} G$ is a ring epimorphic image of the right Artinian ring $R{ }^{*}{ }_{\theta} G$. Therefore $R / J(R){ }_{\sigma} G$ is right Artinian and the coefficient ring $R / J(R)$ is semiprimitive Artinian. Thus $G$ is finite by Lemma 3.2 .

In order to generalize the above theorem to the semiprimary case and perfect case, we start with the following. 
LEMMA 3.4. Let $R$ be $a$ ring and $\theta$ be a group homomorphism from a group $G$ to $\operatorname{Aut}(R)$, and let I be a $G$-invariant ideal of $R$.

(1) If $I$ is nilpotent, then $I *{ }_{\sigma} G$ is nilpotent.

(2) If $I$ is right $T$-nilpotent, then $I{ }^{*}{ }_{\sigma} G$ is right $T$-nilpotent, where $\sigma$ is the group homomorphism from $G$ to $\operatorname{Aut}(I)$ induced by $\theta$.

Proof. (1) Obvious.

(2) Suppose $I *{ }_{\sigma} G$ is not right $T$-nilpotent. Then there is a sequence $\left\{\alpha_{k}\right\}_{k=1}^{\infty}$ in $I *{ }_{\sigma} G$ such that $\alpha_{n} \cdots \alpha_{2} \alpha_{1} \neq 0$ for every positive integer $n$. Therefore for every positive integer $n$, there are elements $a_{1, k(1)}$, $a_{2, k(2)}, \ldots, a_{n, k(n)}$ in $I$ such that $a_{n, k(n)} \cdots a_{2, k(2)} a_{1, k(1)} \neq 0$. Since there is a path of arbitrary length, by Koenig Graph Theorem we conclude that there exists an infinite path: i.e., there exists a sequence $a_{1, k_{0}(1)}, a_{2, k_{0}(2)}, \ldots$ in $I$ such that, for all positive integer $n, a_{n, k_{0}(n)} \cdots a_{2, k_{0}(2)} a_{1, k_{0}(1)} \neq 0$. This contradicts the right $T$-nilpotency of $I$.

LEMMA 3.5. Let $R$ be $a$ ring and $\theta$ be a group homomorphism from a finite group $G$ to $\operatorname{Aut}(R)$. Then $J(R)=R \cap J\left(R *{ }_{\theta} G\right)$.

Proof. That $R \cap J\left(R *{ }_{\theta} G\right) \subseteq J(R)$ follows easily from the definition of the radical. But since $G$ is a normalizing basis of $R *{ }_{\theta} G$ over $R$, we have that $J(R) \subseteq J\left(R *{ }_{\theta} G\right)$ by [9, Theorem 7.2.5, p. 276].

REMARK 3.6. Let $R$ be a ring and $\theta$ be a group homomorphism from a finite group $G$ to $\operatorname{Aut}(R)$. Then by Lemma $3.5, R *_{\theta} G / J\left(R *_{\theta} G\right)$ is a ring epimorphic image of $R *_{\theta} G / J(R) *{ }_{\rho} G$ via the naturally defined ring epimorphism.

Now let us consider the perfect skew group ring case.

THEOREM 3.7. Let $R$ be $a$ ring and $\theta$ be a group homomorphism from a group $G$ to $\operatorname{Aut}(R)$. Then $R *_{\theta} G$ is right perfect if and only if $R$ is right perfect and the group $G$ is finite.

Proof. For the necessity, suppose $R *_{\theta} G$ is right perfect. Then $R$ is right perfect by Lemma 2.2. We note that the technique of the proof for the necessity of Theorem 3.3 can be applied to the perfect skew group ring case by using Lemmas 2.2 and 2.3 in order to reduce the problem down to a right perfect group ring over a field. Then G. Renault's result [11, Theorem 3] or S. M. Woods' result [12] can be applied in order to obtain that $G$ is finite.

For the sufficiency, suppose that $R$ is right perfect and $G$ is finite. Since $R$ is right perfect, $R / J(R)$ is Artinian and therefore $R / J(R) *{ }_{\sigma} R$ is Artinian by Theorem 3.3, where $\sigma$ is the group homomorphism from $G$ to Aut $(R / J(R))$ induced by $\theta$. Therefore $R *{ }_{\theta} G / J\left(R *{ }_{\theta} G\right)$ is right Artinian by Remark 3.6. Let $\pi$ be the canonical homomorphism from $R *{ }_{\theta} G$ to $R *{ }_{\theta} G / J(R) *{ }_{\rho} G$. Then we have that 


$$
\begin{aligned}
\pi\left(J\left(R *{ }_{\theta} G\right)\right) & =J\left(R *{ }_{\theta} G\right) / J(R) *{ }_{\rho} G \\
& \subseteq J\left(R *{ }_{\theta} G / J(R) *{ }_{\rho} G\right)
\end{aligned}
$$

and $J\left(R *_{\theta} G / J(R) *{ }_{\rho} G\right)$ is isomorphic to $J\left(R / J(R) *_{\theta} G\right)$. Hence $J\left(R *{ }_{\theta} G\right) / J(R) *{ }_{\rho} G$ is nilpotent, since it is embedded in $J\left(R / J(R) *{ }_{\sigma} G\right)$ which is nilpotent. Moreover since $J(R)$ is right $T$-nilpotent, $J(R) *{ }_{\rho} G$ is right $T$-nilpotent by Lemma 3.4. Therefore $J\left(R *{ }_{\theta} G\right)$ is right $T$-nilpotent. Hence $R *{ }_{\theta} G$ is right perfect and the proof is complete.

Finally we consider the semiprimary skew group ring case.

THEOREM 3.8. Let $R$ be $a$ ring and $\theta$ be a group homomorphism from a group $G$ to $\operatorname{Aut}(R)$. Then $R *_{\theta} G$ is semiprimary if and only if $R$ is semiprimary and the group $G$ is finite.

Proof. For the necessity, suppose that $R *{ }_{\theta} G$ is semiprimary. Then $R *{ }_{\theta} G$ is right perfect and therefore $G$ is finite by Theorem 3.7. Since $R{ }_{\theta} G$ is right perfect, $R$ is right perfect by Lemma 2.2 and therefore $R / J(R)$ is Artinian. Now since $G$ is finite, we have that $J(R)=R \cap$ $J\left(R *_{\theta} G\right)$ by Lemma 3.5. In this case $J\left(R *_{\theta} G\right)$ is nilpotent because $R *{ }_{\theta} G$ is semiprimary, wherefore $J(R)$ is also nilpotent. Hence $R$ is semiprimary.

For the sufficiency, suppose that $R$ is semiprimary and $G$ is finite. Then again $R *{ }_{\theta} G / J\left(R *{ }_{\theta} G\right)$ is right Artinian and $J\left(R *_{\theta} G\right) / J(R) *_{\rho} G$ is nilpotent as in Theorem 3.8. Since $J(R)$ is nilpotent, $J(R) *{ }_{\rho} G$ is nilpotent by Lemma 3.4. Thus $J\left(R *_{\theta} G\right)$ is nilpotent and hence $R *_{\theta} G$ is semiprimary. The proof is then complete.

4. Semilocal skew group rings. In this section, we will show via an example that the result which we have proved in $\$ 3$ cannot be generalized beyond the perfect case. Also we will characterize those skew group rings over a field which are semilocal.

EXAMPLE 4.1. Let $K$ be an infinite field with characteristic $p>0$ and let $R=\operatorname{Mat}_{2}(K)$. For each $\alpha$ in $K$, define $g_{\alpha}: R \rightarrow R$ such that $g_{\alpha}(A)=$ $\left(\begin{array}{ll}1 & \alpha \\ 0 & 1\end{array}\right) A\left(\begin{array}{cc}1 & -\alpha \\ 0 & 1\end{array}\right)$ for $A$ in $R$. Then $g_{\alpha}$ is a ring automorphism of order $p$. Now let $G=\left\{g_{\alpha}: \alpha\right.$ in $\left.K\right\}$. Then $G$ is locally finite abelian $p$-group and the skew group ring $R * G \cong R[G]$, the ordinary group ring, via the map which sends $A g_{\alpha}$ to $A\left(\begin{array}{ll}1 & \alpha \\ 0 & 1\end{array}\right) g_{\alpha}$. By [4, Theorem 8], $K[G]$ is semiperfect. In [7, Theorem 3] it was shown that a ring is semiperfect if and only if the $n \times n$ matrices over the ring is semiperfect for every positive integer $n$. Hence $R[G]=\operatorname{Mat}_{2}(K)[G] \cong$ $\mathrm{Mat}_{2}(K[G])$ is semiperfect but $G$ is infinite. This example shows us that the semiperfectness or the semilocalness of skew group ring does not imply the finiteness of group when the group $G$ acts faithfully on the ring $R$.

PROPOSITION 4.2. Let $R$ be a semilocal ring and be a group homomorphism from a finite group $G$ to $\operatorname{Aut}(R)$. Then $R *_{\theta} G$ is semilocal. 
Proof. Since $G$ is finite, $R *{ }_{\theta} G / J\left(R *{ }_{\theta} G\right)$ is a ring epimorphic image of right Artinian ring $R / J(R) *{ }_{\sigma} G$ by Remark 3.6. Therefore $R *_{\theta} G$ is semilocal.

Lemma 4.3 (Fisher AND Montgomery [3, Corollary 3]). If $R$ is semiprimitive and $G \subseteq \operatorname{Aut}(R)$ is $\mathscr{F}$-outer, then $R * G$ is semiprimitive.

From this result we obtain the following.

Proposition 4.4. Let $R$ be a semiprimitive ring and $G \subseteq \operatorname{Aut}(R)$ be $\mathscr{F}$ outer. If $R * G$ is semilocal, then $G$ is finite.

Proof. Evident from Theorem 3.3 and Lemma 4.3.

Example 4.1 shows that Proposition 4.4 cannot be established if $G$ is not F-outer.

The following result is an analog of the result [8] for ordinary group ring which states that if $K$ is a field with characteristic 0 and a group ring $K[G]$ is semilocal, then $G$ is finite. However, it is not necessary to assume the characteristic of the coefficient field is zero in the skew group ring case if we assume $G$ acts faithfully on $K$.

Proposition 4.5. Let $K$ be $a$ field and $G \subseteq \operatorname{Aut}(R)$. Then the following are equivalent.

(1) $K * G$ is semiperfect.

(2) $K * G$ is semilocal.

(3) $G$ is finite and $K * G \cong \operatorname{Mat}_{n}\left(K^{G}\right)$, where $K^{G}$ is the fixed field under the action of $G$ and $n$ is the order of $G$.

Proof. That $(1) \Rightarrow(2)$ and $(3) \Rightarrow(1)$ are evident.

$(2) \Rightarrow(3)$. By Proposition 4.4, $G$ is finite. Now let $n$ be the order of $G$. Then by [6, Lemma 4.4.2, p. 111], we have that $K * G \cong \operatorname{Mat}_{n}\left(K^{G}\right)$.

ACKNOWLEDGEMENT. The author wishes to express his deepest appreciation to Dr. Joe W. Fisher and Dr. James Osterburg for their helpful advice and encouragement during preparation of this paper. Also, the author is grateful to the referee for helpful comments and for suggesting Lemma 3.4.

\section{REFERENCES}

1. F. Anderson and K. R. Fuller, Rings and categories of modules, Springer-Verlag, Berlin, Heidelberg and New York, 1974.

2. I. G. Connell, On the group ring, Canad. J. Math. 15 (1963), 650-685.

3. J. W. Fisher and S. Montgomery, Semiprime skew group rings, J. Algebra 52 (1978), 241-247.

4. J. M. Goursaud, Sur les anneaux de groupes semi-parfaits, Canad. J. Math. 25 (1973), 922-928.

5. D. Handelman, J. Lawrence and W. Schelter, Skew group rings, Houston J. Math. 4 (1978), 175-198.

6. I. N. Herstein, Noncommutative rings, Carus Math. Monographs, no. 15, Math. Assoc. Amer., Wiley, New York, 1968.

7. S. Keye, Ring theoretic properties of matrix rings, Canad. Math. Bull. 10 (1967), 365-374. 
8. J. Lawrence and S. M. Woods, Semilocal group rings in characteristic zero, Proc. Amer. Math. Soc. 60 (1976), 8-10.

9. D. S. Passman, The algebraic structure of group rings, Wiley, New York, 1977.

10. __ Radical of twisted group rings. II, Proc. London Math. Soc. 22 (1971), 633-651.

11. G. Renault, Sur les anneaux de groupes, C. R. Acad. Sci. Paris Sér. A-B 273 (1971), A84-A87.

12. S. M. Woods, On perfect group rings, Proc. Amer. Math. Soc. 27 (1971), 49-52.

Department of Mathematics, University of Cincinnati, Cincinnati, Ohio 45221

Current address: Department of Mathematics, Busan National University, Busan, Korea 607 